\title{
Internet based light quality measurement
}

\author{
Krisztián Samu ${ }^{1}$, Bálint Thamó ${ }^{2}$ \\ Department of Mechatronics, Optics, and Engineering Informatics \\ BME, Budapest University of Technology and Economics \\ Budapest, Hungary \\ 1samuk@mogi.bme.hu, b.thamo@gmail.com
}

\begin{abstract}
The goal of this project was to implement a device, which can measure the illuminance and the correlated color temperature (CCT) of a light source and also works as a webserver. The analyzation of light sources is very important in our everyday life. With the use of a webserver the measured values are easily accessible and the calculation methods can be changed without any changes on the hardware. It also provides a solution to store and analyze the results without further human interaction. Therefore the device is capable of collecting and analyzing a large amount of data. For these criterions we chose a PIC microcontroller, an Ethernet chip and two different sensors. One of the sensors is an RGB sensor and the other one is a LUX sensor. Both of the sensors are using photodiodes to sense the different attributions of a light source. Through this article we show the basic theory behind the project, the implementation and calibration of the device; then the results of the measurements and finally we mention some possibilities for future improvements.
\end{abstract}

Keywords- webserver; correlated color temperature; illuminance; sensor; microcontroller; light source; data collection; calibration; measurement; photodiode; chromatical diagram; planckian locus

\section{INTRODUCTION}

Analyzing light sources is very important in our everyday life and we usually do not pay enough attention to select the proper light sources. A light source could affect our wellbeing, working quality and emotions. The workplaces usually take care about the appropriate light sources but people in general are not concerned with this topic. Selecting adequate light sources at home, it is possible to improve our quality of life. The two main attributions of light sources are the illuminance and the correlated color temperature (CCT), which provide enough information for us to analyze them and select an appropriate one. Laboratory is a key element of engineering and applied sciences educational systems. With the development of Internet and connecting IT technologies, the appearance of remote laboratories was inevitable. Virtual laboratories are also available; they place the experiment in a simulated environment. However, this writing focuses on remote experiments not virtual ones. An example of remote experiments at our Department is aimed at allowing students to remotely control ethologically inspired robot [1], [2] and a servo motor [3]. The student can learn the theoretical background by animations [4], [5]. Since the appearance of remote laboratories many architectures were designed. New tools and trends have always been built into these designs. Currently, the fastest developing technology is HTML5 and cloud based remote laboratories and for that, remote desktop technologies provide perfect solutions. For example, with Guacamole, in a HTML5 enabled web browser a remote desktop can be displayed without any additional plugin. Also HTML5 supports portable devices such as smartphones and tablets and this accelerates the spread of remote experiments. This technology was applied to develop the Internet based light quality measurement.

\section{THEORY}

The Illuminance is the amount of light energy reaching a given point on a defined surface area. The unit for illuminance: lux. [6], [9], [11]

The color temperature refers to the temperature to which one would have to heat a blackbody radiator to produce light of a particular color. [10], [12]

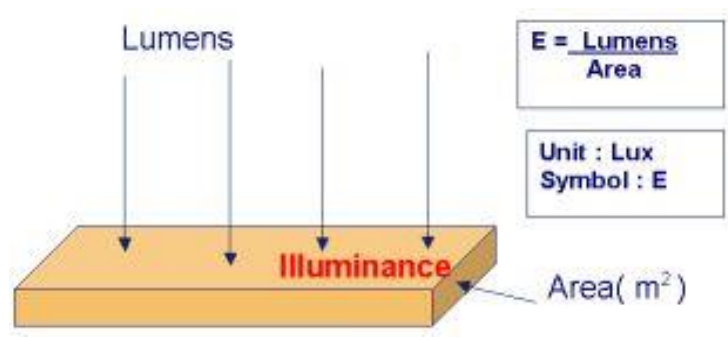

Fig. 1 Illuminance definition [8]

A blackbody radiator is defined as a theoretical object that is a perfect radiator of visible light. As the blackbody radiator is heated, it radiates energy first in the infrared spectrum and then in the visible spectrum as red, orange, white and finally bluish white light. Although incandescent lights are good models of blackbody radiators, most of the light sources are different from it, so we have to find a method to somehow relate them to it. [12]

To do so, the use of Correlated Color Temperature (CCT) is necessary. CCT is defined as the temperature of a blackbody radiator whose chromaticity point is the closest to the chromaticity point of the non-planckian light source. The planckian locus on the chromatical diagram shows the different color temperatures in a special color space, called CIE1932 
color space. The closer the point to the planckian locus, the more accurate the result is. Both the color temperature and the correlated color temperature are measured in Kelvin. [7], [14]

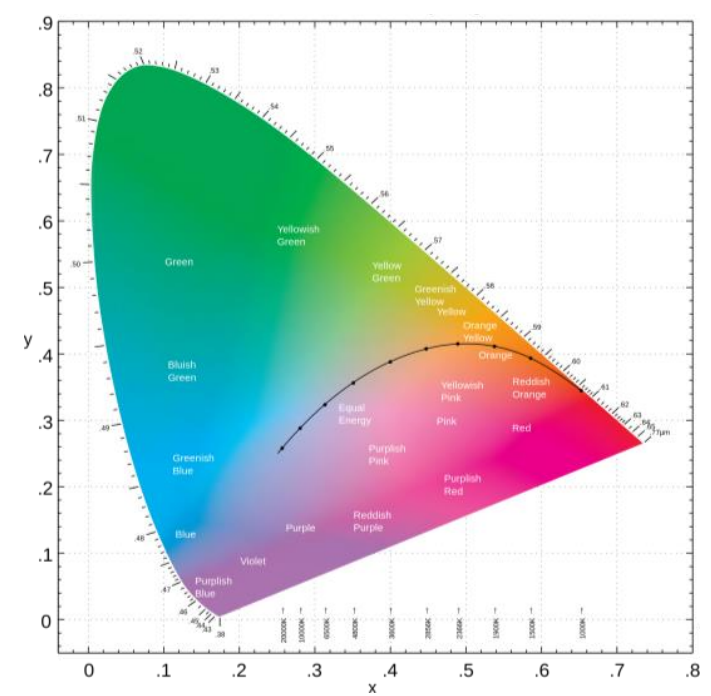

Fig. 2 The CIE 1931 Chromaticity Diagram [6]

\section{IMPLEMENTATION OF THE DEVICE S}

The most important criterions for the device are the accurate CCT, illuminance measurement and access through the internet. From the possible solutions the photodiode fitted to our needs the best because it was quite accurate and also had a reasonable price. But above all it was the most obvious choice for a webserver. Therefore we picked an RGB sensor and a Lux sensor made by Adafruit. The RGB sensor has the TCS34725 color light-to-digital converter and the Lux sensor has TSL2561 light-to-digital converter from TAOS. Both of the sensors have infrared filters and communicate over I2C. To communicate with the sensors, we chose a PIC18F46k80 microcontroller from Microchip. In this way the microcontroller is capable to communicate with the sensors and give results via USART communication. Moreover, to make it also work as a webserver, the system has an ENC28J60 Ethernet chip which is capable of communication via Ethernet. The chip can communicate with the microcontroller via SPI. Having chosen this configuration it is possible to write a basic HTML code to the source code of the microcontroller and make it work as a webserver. However, the microcontroller has little memory, so in order to do more complex calculations, the device sends its measured values from the of the microcontroller to a PHP page via POST and GET methods.

After this it is possible to do more complex calculations on more complex web page or on our computer with the collected data.

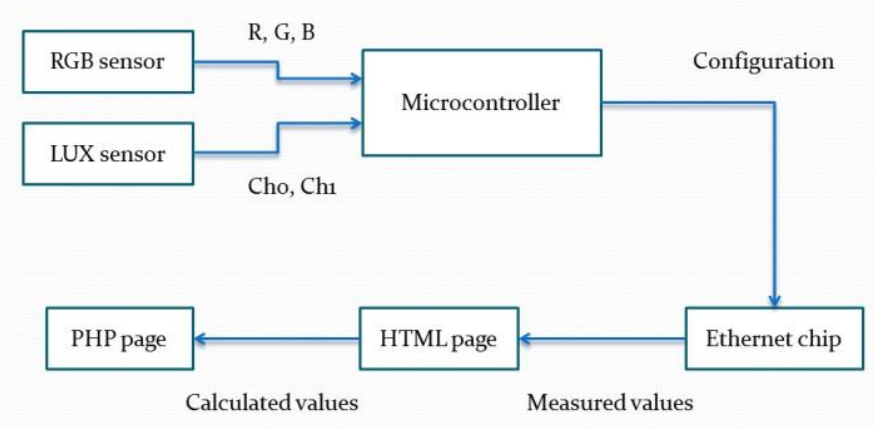

Fig. 3 Flow chart of the device

\section{SENSORS}

The Lux sensor has two photodiodes. One of the photodiodes is sensible for the visible light and for the infrared light (Channel0). The other one is only sensible for the infrared light (Channel1). The difference between the two photodiodes is similar to the sensibility of the human eye $(V(\lambda))$, therefore this sensor is suitable for our device [9].

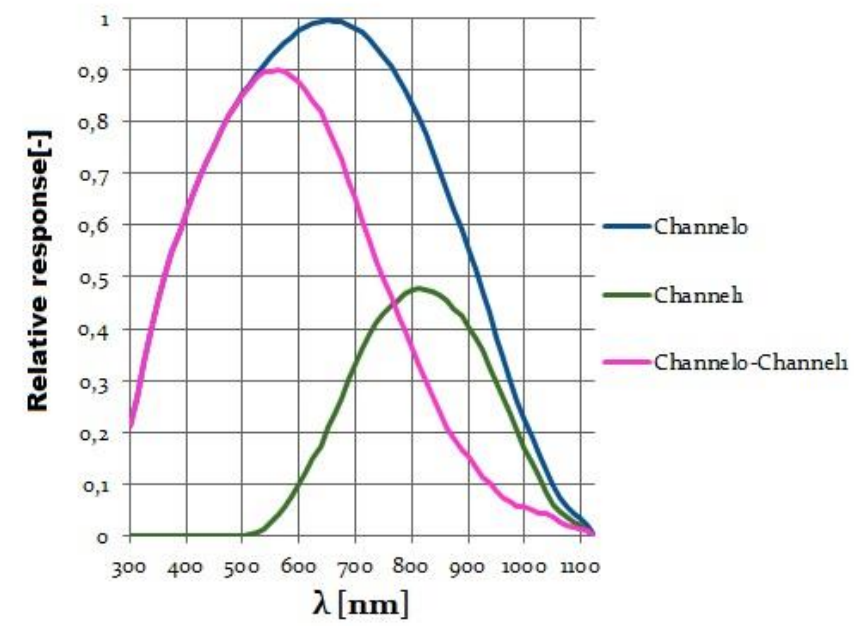

Fig. 4 Sensitivity of the LUX sensor [16] 
The RGB sensor provides the red, blue and green content of a light source; it contains three photodiodes with different filters for each color. It has a broad scale as it measures the values on 16 bits [15].

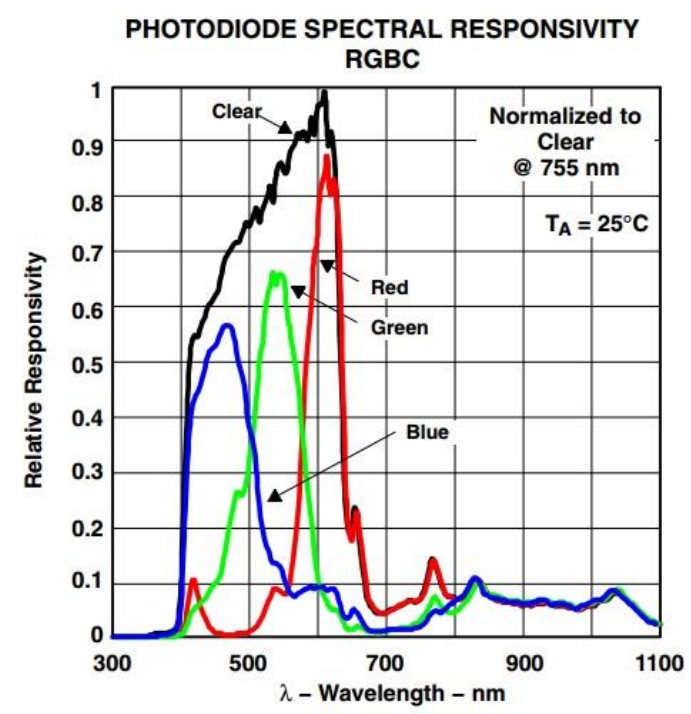

Fig. 5 Sensitivity of the RGB sensor [7]

However, these values must be transformed to standard tristimulus values $(\mathrm{X}, \mathrm{Y}, \mathrm{Z})$ because it is not possible to calculate the CCT from the RGB coordinates without any transformation. This transformation is a matrix multiplication (1-2), where the elements of the correlation matrix (C) are from a reference measurement from Commission Internationale de l'Eclairage (CIE). The other two matrixes are containing the tristimulus values (T) and the measured values by the sensor (S). From the tristimulus values we have to calculate the chromaticity coordinates to get the above mentioned color space (0) where the planckian locus can be displayed. Finally, the CCT can be calculated from the chromaticity coordinates with the help of the form of McCamy (5-6). This form provides an error less than 2 percent for CCT calculation. The illuminance can be also calculated from the transformed R, G, $B$ values where the illuminance is equal to the Y-value of the standard tristimulus values. [12], [13]

$$
T=C \cdot S
$$

$$
\begin{aligned}
& {\left[\begin{array}{l}
X \\
Y \\
Z
\end{array}\right]=[S] \cdot\left[\begin{array}{l}
R \\
G \\
B
\end{array}\right]} \\
& x=\frac{X}{X+Y+Z} \\
& y=\frac{Y}{X+Y+Z}
\end{aligned}
$$

$$
\begin{gathered}
n=\frac{x-0.3320}{0.1858-y} \\
\mathrm{n}=449 \cdot n^{3}+ \\
+3525 \cdot n^{2}+ \\
+6823.3 \cdot n+ \\
5520.33
\end{gathered}
$$

\section{CAlibration}

At this stage, the device is already capable of measuring but the measured values were not accurate enough, therefore calibration was necessary.

For the CCT calibration a spectrophotometer was the most obvious solution. This device was calibrated with a Spectra Spectrophotometer. For the calibration a new correlation matrix (C) was created (8) where T matrix contains the $\mathrm{X}, \mathrm{Y}, \mathrm{Z}$ and $S$ matrix contains the R, G, B values of three different lights sources. All values were also measured with a reference device. As we wanted to, the calibration made it possible indeed to get more accurate results for specific light sources. [7], [17]

$$
C=T \cdot S^{-1}
$$

For illuminance calibration there are more available solutions, among which the use of a lux meter is the most common solution as a reference. Therefore, the device was calibrated with a Voltcraft MS-1300 lux meter. For the calibration we used a light source with a changeable intensity. We set the intensity of the light source from $10 \%$ to $100 \%$, increasing the intensity by $10 \%$ each time, and took a measurement in each step. After this, a constant multiplier was calculated between the lux meter and the device.

\section{RESULTS}

After calibrating the device the error of the measured values for CCT were less than 7 percent comparing to the reference spectrophotometer as shown is Fig. 66.

For illuminance the error was less than 3.5 percent with the LUX sensor and less than 5 percent with the RGB sensor comparing to the reference lux meter as shown is Fig. 77. 


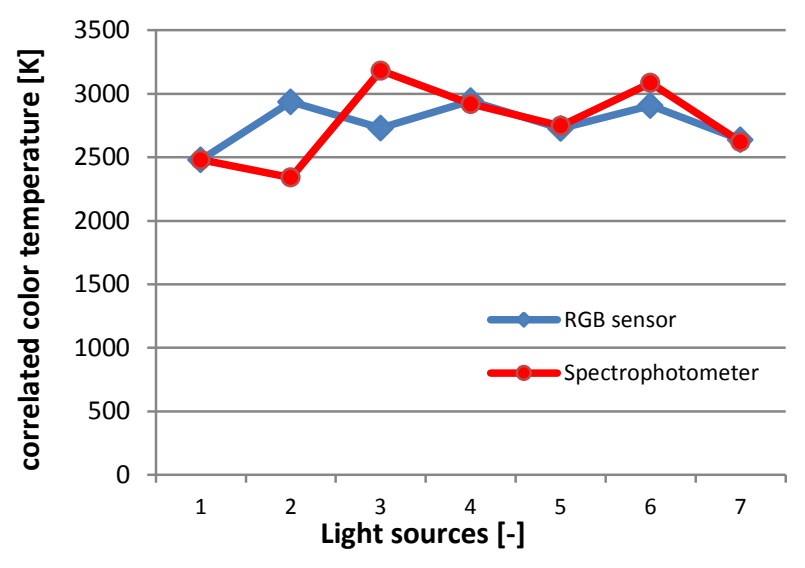

Fig. 6 Measured results for CCT

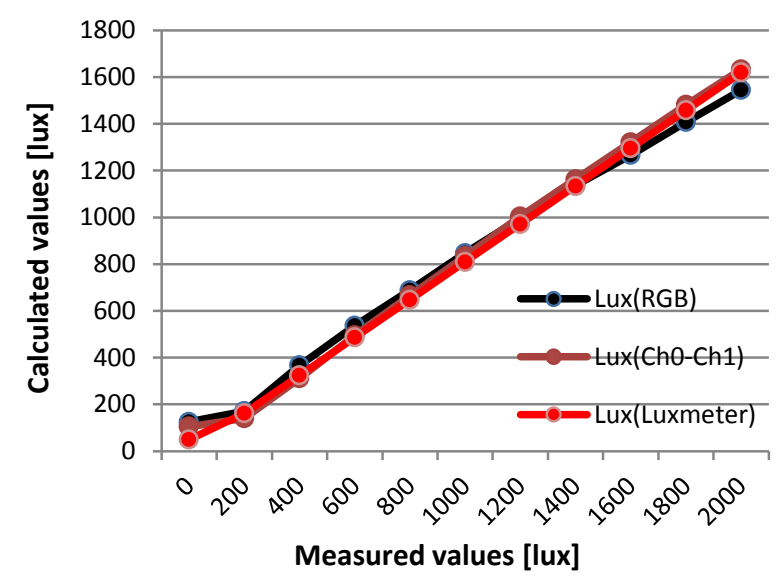

Fig. 7 Measured results for illuminance

This result is satisfactory but can be improved. If we know what kind of light source we would like to measure, then it would be possible to make the calibration more specific or to make some modifications in the source code of the microcontroller to get more accurate results.

\section{CONCLUSION}

The device is capable of measuring the illuminance and correlated color temperature of a light source in real time. The calculated values are accurate enough for general usage but not for all kind of light sources. However, to make it more efficient, we can analyze the measured data and decide the desired light sources what we want to measure. The device can be accessible through the internet which makes it easy to collect a large amount of data from the microcontroller. One can also make changes in the calculations easily because the microcontroller only reads the values from the two sensors and then sends them directly to a web server where it is possible to make a new calibration method or change the steps of the calculations. In the future, a learning algorithm could be used from the collected data to make the calibration and calculation more accurate to specific light sources.

\section{ACKNOWLEDGMENT}

The Emerging Information Technology - Development of training modules project (HU08-0007- M4) benefits from a grant from Iceland, Liechtenstein and Norway through the EEA Grants and Norway Grants (EGT/156/M4-0004). The aim of the project is to develop useful, modern teaching and Internet-based laboratory measurements on the field of Robotics, Metrology, Biomechatronics and Energetics that meets requirements of industry.

\section{REFERENCES}

[1] B. Nagy, M. Papp,L. Raj, R.T. Fekete, "Development of a behavior engine in ethorobotics system and implementation on a mobile robot in LabVIEW environment", in Proceedings of the International Symposium on Small-scale Intelligent Manufacturing Systems (SIMS). Narvik, Norway. pp. 121-125, 2016

[2] R.T. Fekete, B. Korcsok, L. Raj, "Affective psychological basis of behavioural modelling and simulation in ethorobotics", in Proceedings of the CERiS'15: Workshop on Cognitive and Etho - Robotics in iSpace, Budapest, Hungary, pp. 30-39,2015

[3] G Sziebig, B Takarics, P Korondi, "Control of an embedded system via internet", in IEEE Transactions on Industrial Electronics 57 (10),pp. 3324-3333, 2010

[4] V. Fedak, P. Bauer,V. Hájek,H. Weiss, B. Davat,S. Manias, I. Nagy, P. Korondi, R. Miksiewicz, P. Duijsen, P. Smékal, "Interactive E-Learning in Electrical Engineering", in Proceedings of the 15th International Conference on Electrical Drives and Power Electronics (EDPE), Podbanské, pp. 368-373, 2003

[5] H. Weiss, A. Schmidhofer, A. Schmid, V. Hajek, B. Davat, S. Manias, I. Nagy, P. Korondi, K. R. Jardan, R. Miksiewicz, V. Fedak, P. Smekal, P. Bauer, P. van Duijsen, "Animated and Interactive E-Learning Concept and Realization," in Proceedings of the IASTED International Conference on Web-Based Education (WBE), 2004

[6] CIE: International Lighting Vocabulary, CIE S 017/E, ISBN 9783900734077, 2011

[7] G. N. Brazhnichenko: Exact equations for calculation of color temperature of hot bodies High temperature, Vol. 14(2), pp: 384-386, 1976

[8] EMS Production Ltd. http://www.hkems.com/LED/images/QA/Illuminance.jpg [Online]. Available: 01.06.2016.

[9] D. B. Judd: 1900-1972.Contributions to color science, National Engineering Laboratory, National Bureau of Standards, 1979

[10] D. L. Macadam: Correlated color temperature. JOURNAL OF THE OPTICAL SOCIETY OF AMERICA, Vol. 67(6), pp: 839-840, 1977

[11] D. Malacara: Color Vision and Colorimetry Theory and Applications, SPIE Press, Bellingham WA, 2002

[12] C. McCamy, "Correlated color temperature as an explicit function of chromaticity coordinates," Color Research \& Application, pp. 142144,1992

[13] M. Mitsunori; M. Yo; Y. Kenta; et al.: A Prototype of Digital Illuminance Sensor for The Distributed Control Lighting System. Proceedings of the 2013 IEEE conference on Cybernetics and Intelligent Systems (CIS) Pages: 90-95, Manila, PHILIPPINES, 2013

[14] J. Smith: Calculating Color Temperature and Illuminance. TAOS INTELLIGENT OPTO SENSOR - DESIGNER'S. NOTEBOOK No. 25,2009 
Recent Innovations in Mechatronics (RIiM) Vol. 4. (2017). No. 1. DOI: $10.17667 /$ riim.2017.1/4

[15] TAOS: TCS34725 Datasheet, https://www.adafruit.com/datasheets/TCS34725.pdf, 2012.

Vision, Bogota, 2013

[16] TAOS TSL2560 Datasheet, https://cdnshop.adafruit.com/datasheets/TSL2561.pdf, 2009.

[17] J.-S. B. Valencia, F.-E. L. Giraldo, J.-F. V. Bonilla: Calibration method for Correlated Color Temperature (CCT) measurement using RGB color sensors. XVIII Symposium of Image, Signal Processing, and Artificial 